\title{
Laser method of sound emission control from vibrating surfaces of noise road barriers
}

\author{
Zbigniew Motyka \\ Central Mining Institute, Katowice, Poland \\ E-mail: zmotyka@gig.eu \\ Received 7 July 2017; accepted 11 July 2017 \\ DOI https://doi.org/10.21595/vp.2017.18840
}

Check for updates

\begin{abstract}
Proposition of a new hybrid measurement method applying for road acoustic barriers infrastructure maintenance, planning and development on the base of innovative vibro-acoustic control of their efficacy, is presented and discussed. The method is based on an interdisciplinary approach using optical LDV, computational and acoustic methods.
\end{abstract}

Keywords: road noise barriers, laser Doppler vibrometry, virtual modelling.

\section{Introduction}

Due to rapid development of the particular road infrastructure which are noise road barriers, the necessity arises of developing of new complex and possibly universal assessment and planning system for the noise barriers replacement and development, but also removing them when used and unnecessary, including final waste management and possible recycling. Such system of standardized procedures and processing would start from both in situ measurements as well as virtual simulations. Therefore, it will become useful already at the assessment stage of already existing barriers. Also, it may be used for determining the necessity of applying modernized, new designed noise road barriers. In consequence, it would lead toward more effective planning and scheduling of necessary measures on the development stage. It could be also achieved by formulation of validated indices for the necessary measures targeted to creation of friendlier for environment, as much as possible converging toward future noise-free green road transporting system.

To achieve this, the new hybrid method is proposed involving the interdisciplinary optical, computational and acoustic methods focused on application of laser measurements of surface vibrations for determining and visualization of acoustic field generated by the vibrations in surrounding. The Laser Doppler Vibrometry (LDV) methods [1] will be used for determining the parameters of existing noise barriers, including both their efficiency in road noise dumping and assessment of degree of their deterioration. The sound transmission through them will be assessed selectively by determining their input to noise level from vibrations induced within their body by road traffic. The transmitted sound level will be calculated on the base of the data from LDV. This will be achieved with the use of specialized procedures, including some of them already developed in GIG and described in the paper. On the base of this way obtained results and standard noise measurements, the separation will become possible of noise barrier surface vibration input from the noise input of acoustic waves transmitted over the barrier.

There are many methods of direct experimental visualization of space distribution of acoustic field. Intensity sound probes [2-7], their arrays and based on them near-field acoustic cameras [8], acoustic holography [9-11], or beamforming [12-14] are examples of such methods. Each of them can be also adapted as complementary tool for presented method verification. The proper selection of such tool will depend on the range of conducted tests, and will enable the verification of appliance scope, as well as calibration and validation of the developed methodology. Its final form would enable to assess the quantitative input of acoustic barriers surfaces to the level of road noise emitted to environment on the base of computer analysis of these surfaces vibrations. The Microflown PU sound probes and based on them acoustic cameras form the worlds unique MEMS technology based sensors, that can measure the acoustic particle velocity. This extremely sensitive and fast mass flow sensors are capable of monitoring the movement of air particles by measuring 
the temperature difference in the cross section of two extremely thin platinum wires placed in parallel. And as any sound field is described completely by both the (scalar) value sound pressure and the (vector) value acoustic particle velocity, acoustic testing becomes much easier if both the acoustic quantities can be measured. These and other possible methods can be used to calibrate and validate the developed methods of virtual assessment of differentiated input from (both deteriorated as well as still in good condition) panels of noise barriers with sound transmitted through, or even additionally generated by them, from the sound transmitted over the barrier. The measured data on both compounds will be then used for modeling of newly planned and being developed road noise barrier systems with the use of advanced digital acoustic methods calculations, such as provided, e.g., by LMS Virtual.Lab software.

\section{Determining of acoustic field distribution from simulated picture of surface vibrations}

Both vibration of plane surfaces and sound field induced by them in surrounding air may be calculated with the use of LMS Virtual.Lab software for suitably developed models. Fig. 1. presents an example of vibrations calculated for model vibrating surface, obtained with the use of LMS Virtual.Lab. Fig. 2. presents an example of acoustic field calculations results obtained within LMS Virtual.Lab Acoustics in the open space adherent to simulated model vibrating surface.

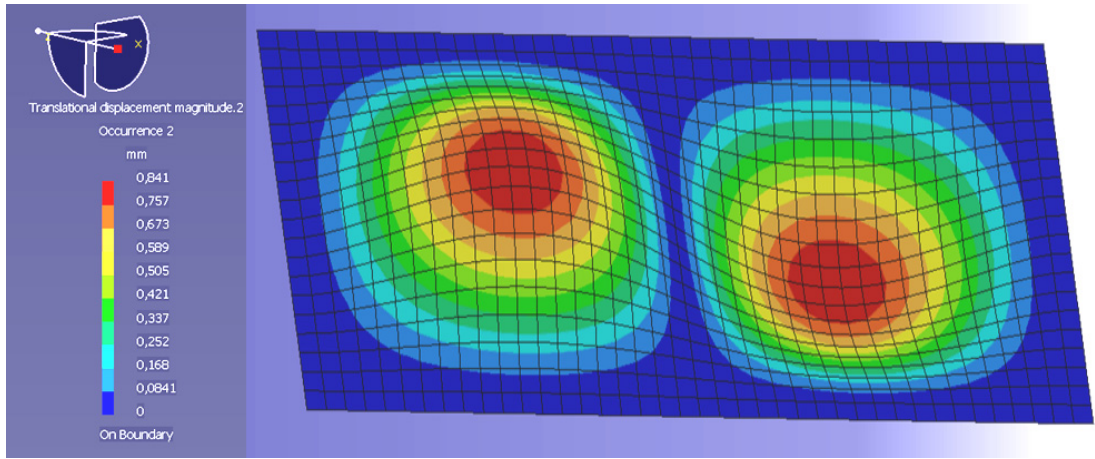

Fig. 1. The second mode of vibrations of model vibrating surface obtained by author with the use of LMS Virtual.Lab

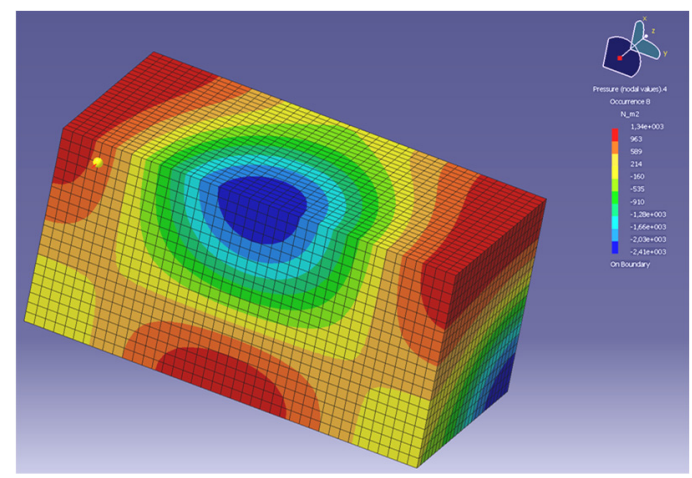

Fig. 2. An example of acoustic field calculated by author with the use of LMS Virtual.Lab Acoustics in the direct vicinity of modeled vibrating surface

\section{Determining of acoustic field distribution from true picture of surface vibrations}

Modern laser measurement techniques enable to determine true vibrations of relatively very complex surfaces. It means that, for true, existing surfaces, experimental determining of amplitudes and phases of particular elements of these surfaces is possible without necessity of 
conducting complex digital simulations. From the amplitude distributions of tested surfaces vibrations obtained from LDV measurements, the simplified digital calculations are possible of sound intensity and acoustic pressure in selected points nearby these surfaces. To perform this task, the modified hybrid method may be used [15-18]. Apart of vibration magnitude for each of small square elements of analyzed surface, also the distribution of relative phase vibration of all these particular square elements between themselves have to be considered. This is possible with the use of LDV method which enables to obtain such phase vibration distributions for elements of tested surface. The proper data will be obtained when assuring maximal dimension of such an element to be much smaller than the transverse elastic wave velocity within the material of tested plane divided by its angular frequency.

The acoustic field pressure $\mathrm{p}$ and velocity $\mathbf{v}$ at any point $\mathrm{P}$ in free space over such a surface can be calculated as a sum of such inputs $p_{i}$ and $\mathbf{v}_{i}$ from all the finite square elements and may be determined analytically and calculated digitally (the detailed way of obtaining this solution was presented in the paper [18]):

$p=\sum_{i} p_{i}, \quad \mathbf{v}=\sum_{i} \mathbf{v}_{i}$.

The resulting time averaged acoustic field intensity at $\mathrm{P}$ is:

$I=\frac{1}{2} \operatorname{Re}\left(p \mathbf{v}^{*}\right)$.

In such a way it may be selectively determined in situ for sound transmitted/emitted only by the screen surface. Therefore, an interference from sound traveling over the screen is omitted here, enabling assessment of true screen condition. Normally, this is possible with the use of traditional methods only in laboratory conditions of anechoic chambers and for very restricted experimental setups.

Fig. 3. presents acoustic field pressure levels calculated by the above hybrid method for frequency $25 \mathrm{~Hz}$ in parallel planes distanced by 5 and $45 \mathrm{~cm}$, respectively, from small $18 \mathrm{~cm}$ square metal model plane vibrating surface. They were calculated on the basis of true phase and amplitude data on vibrations of particular elements of this surface obtained with the use of LDV method.

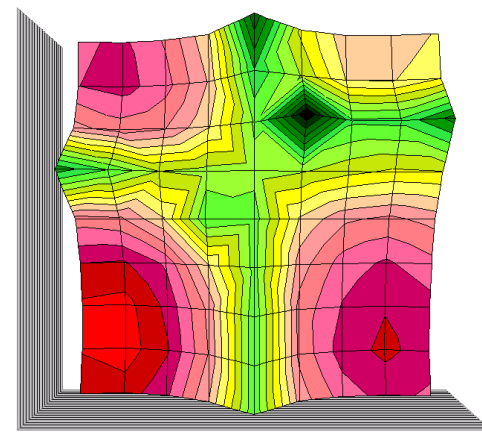

a) $5 \mathrm{~cm}$

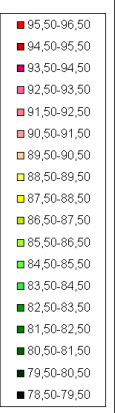

$8,50.79,50$

Fig. 3. Acoustic pressure levels [dB], obtained by author for the case of sound wave of frequency $25 \mathrm{~Hz}$ emitted by small plane vibrating surface, calculated at the distance of $5 \mathrm{~cm}$ and $45 \mathrm{~cm}$. The vertical axes of acoustic pressure levels at these $3 \mathrm{D}$ perspective plots are directed behind the page plane

\section{Traffic induced surface vibrations of plane panels of road acoustic barriers}

Analysis of vibrations of existing road acoustic barriers induced by road traffic is possible after 
in situ measurements, performed, e.g., with the use of Laser Doppler Vibrometry [19].

The surface of chosen single panel (or its part) can be divided into the rectangular lattice of elementary squares (Fig. 4(a)). In situ measurements of low frequency vibration amplitude for the nodes of such lattice were performed with the use of PSV-400 Laser Vibrometer (Fig. 4(b)).

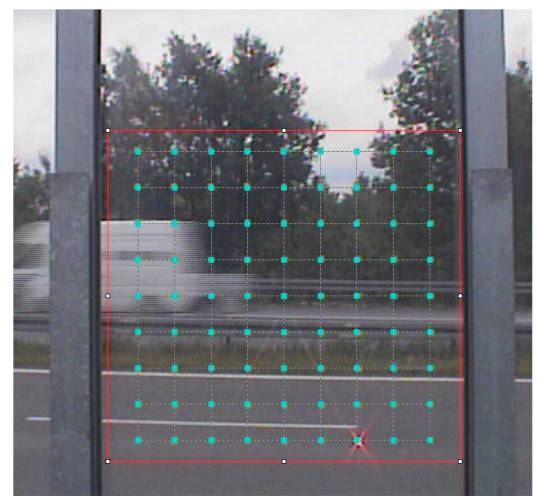

a)

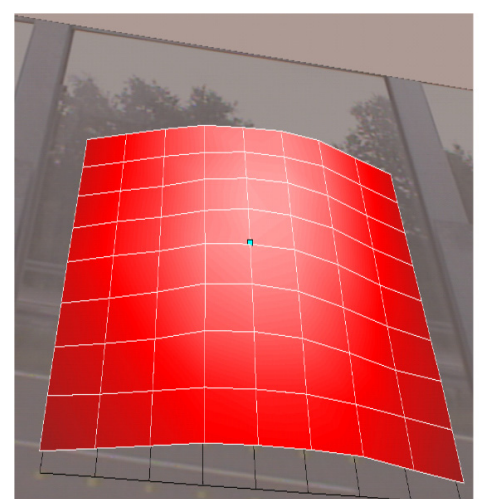

b)

Fig. 4. a) Panel of noise barrier with measurement lattice on LDV screen captured during "in situ" measurements of vibrations, and b) an example of the road noise barrier panel vibrations obtained by author for frequency $0.75 \mathrm{~Hz}$

\section{Virtual designing of road acoustic barriers layout}

Simulation of efficiency of fully developed noise road barriers infrastructure in the form of series of noise screening panels is possible for data obtained for one only or few typical such panels. Both for already existed (for comparison and validation needs), as well as still being designed to build up constructions. In the latter case, it means the possibility of building advanced predictive models of series of noise barrier panels to be build up. Such models, composed from particular sections/panels, in general of different types, may form long curved road acoustic barriers following the road curvature (Fig. 5).

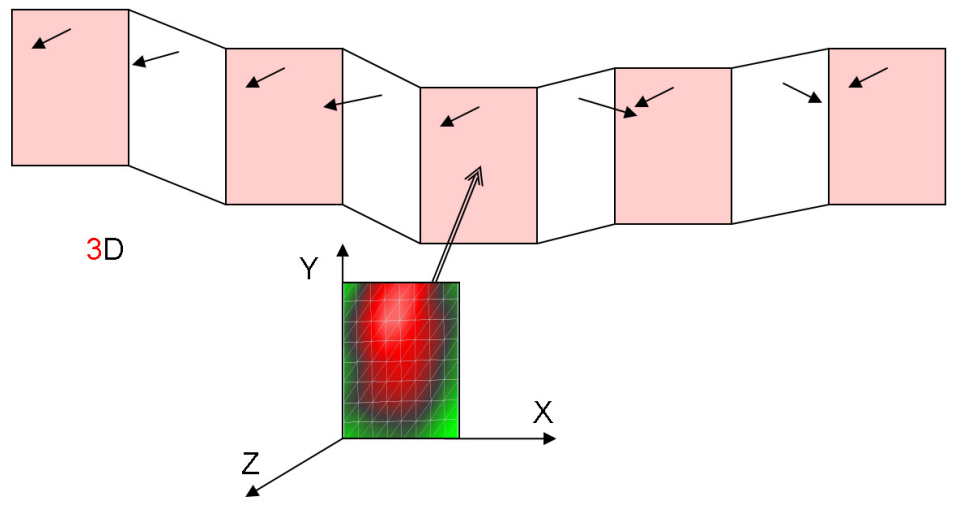

Fig. 5. Scheme of simulation of space layout of rectangular noise screens forming noise barrier following curvature of the road and adjacent terrain. The LDV vibration data for a single screen have to be transformed to many copies in 3D layout, for which the sound field is calculated with the use of LMS Virtual.Lab

The analogue of Cóser scheme may be adapted here, who simulated a spherical loudspeaker array using a flexible membrane model for single-loudspeaker [20]. The LDV data for a single membrane, were transformed by him to many copies in 3D layout for which the sound field was 
calculated with the use of LMS Virtual.Lab. In a similar way, the scheme from Fig. 5. of simulation of space layout of rectangular noise screens forming noise barrier following curvature of the road may be used. The LDV data for a single noise road panel have to be transformed to many copies in 3D layout, for which the sound field can be calculated with the use of LMS Virtual.Lab. Such an approach, through innovative virtual modeling of noise road barriers infrastructure efficacy, will enable effective enough planning of their development.

When applying Eqs. (1) and (2) for all elementary components of all duplicated this way panels, the resulting acoustic field in any point in the space adherent to the noise road barrier may be also calculated by means of the more sophisticated hybrid method, as the latter enables also considering of different patterns of phase dependencies among the linear series of panels within the barrier resulting from different modeling of traffic intensity. It could give precise information on noise levels depending on different traffic character, and could enable more precise planning taking into account also the different conditions in different time of a day only on the basis of measured (or simulated) traffic patterns.

\section{Conclusions}

The proposed innovative approach for effective planning and designing of complex long noise road barriers infrastructure may enable development of virtual modeling of their efficacy on the basis of "in situ" measurements for single panels representative for planned construction. The particular elements of proposed method were tested both for simulated, as well as true objects, forming firm basis for its applying for the true road barrier infrastructure.

This approach assumes use of data obtained for such representative types of panels based on their surface vibrations in situ measurements with optical LDV method. They can be multiplied virtually accordingly to modeled structure of planned noise road barrier composed of such panels. In such a way, taking the true characteristics of terrain and road curvature into account at such modeling, one can selectively obtain acoustic field transmitted by the noise barrier to the environment in the direct vicinity of the road, using such convenient tool as, e.g., LMS Virtual.Lab. Moreover, the hybrid method of calculations, when applied to the whole structure, will enable to take into account also phase dependencies from the traffic pattern.

The geometry of such model may also enable in the future independent modeling of input to acoustic field from sound waves passing over the noise barrier, on the base of calibration measurement for each panel type. However, to achieve this aim an additional solver Ray noise of Acoustic module of LMS Virtual.Lab software packet has to be used. The possibilities of its usefulness have to be subject of another study, though. If its usefulness would be confirmed, then both the components could be used for calculations of complete virtual noise maps in the vicinity of the roads.

\section{References}

[1] Castellini P., Martarelli M., Tomasini E. P. Laser doppler vibrometry: development of advanced solutions answering to technology's needs. Mechanical Systems and Signal Processing, Vol. 20, Issue 6, 2006, p. 1265-1285.

[2] Weyna S. Application of microflown probe to visualization of acoustic power flow. Scandinavian Structured Conference on Acoustic, Poznan-Wagrowiec, Poland, 2005, (in Polish).

[3] Weyna S. Microflown based identification of vortex shadding in the space of real acoustic flow fields. ICSV12, Lisbon, 2005.

[4] Mickiewicz W., Jabłoński M. J. Automatised system for spatial visualisation of acoustic field. Pomiary Automatyka Kontrola, Vol. 58, Issue 11, 2012, p. 920-922.

[5] Pohl M., Rose M., Haase T. Actuator placement for shunt damping of panel structures - numerical simulation and experimental validation. International Conference on Adaptive Structures and Technologies, Aruba, 2013. 
[6] Yntema D. R. An Integrated Three-Dimensional Sound-Intensity Probe. Ph.D. Thesis, University of Twente, Enschede, The Netherlands, 2008.

[7] De Bree H. E., et al. The microflown; a novel device measuring acoustic flows. Sensors and Actuators: Physical A, Vol. 54, Issues 1-3, 1996, p. 552-557.

[8] De Bree H. E. A Multi purpose sound intensity based acoustic camera. Conference and Exposition on Structural Dynamics (IMAC-XXIV), 2006, p. 1493-1498

[9] Jacobsen F. Sound Intensity and its measurement and applications. Acoustic Technology, Rsted DTU, Technical University of Denmark, Note No. 31262, 2005.

[10] Jacobsen F., Liu Y. Near field acoustic holography with particle velocity transducers. The Journal of the Acoustical Society of America, Vol. 118, Issue 5, 2005, p. 3139-3144.

[11] Jacobsen F., Jaud V. Statistically optimized near field acoustic holography using an array of pressure velocity probes. The Journal of the Acoustic Society of America, Vol. 121, Issue 3, 2007, p. $1550-1558$.

[12] Cristensen J. J., Hald J. Technical Review, Beamforming. Bruel \& Kjaer, 2004.

[13] Michel U. History of acoustic beamforming. 1st Berlin Beamforming Conference (BeBeC), 2006.

[14] Haxter S., Spehr C. Infinite beamforming: wavenumber decomposition of surface pressure fluctuations. Berlin Beamforming Conference, 2014.

[15] Kozień M. S. Hybrid method of evaluation of sounds radiated by vibrating surface elements. Journal of Theoretical and Applied Mechanics, Vol. 43, 2005, p. 119-133.

[16] Kozień M. S. Acoustic intensity vector generated by vibrating set of small areas with random amplitudes. Journal of Theoretical and Applied Mechanics, Vol. 47, 2009, p. 411-420.

[17] Motyka Z. The hybrid method of laser monitoring of acoustic emissions from vibrating surfaces. Wiadomości Górnicze, Vol. 6, 2010, p. 364-368.

[18] Motyka Z. Laser method of acoustic emission control from vibrating surfaces. Proceedings of SPIE, 2013.

[19] Bartmański C., Motyka Z., Staniek A. et al. Analysis of acoustic road barriers vibrations with the use of LMS Test.Lab software. Pomiary Automatyka Kontrola, Vol. 60, Issue 12, 2014, p. 1100-1103.

[20] Cóser L. F. Simulation of a Spherical Loudspeaker Array Using a Flexible Membrane Model. M.Sc. Thesis in Mechanical Engineering, Faculty of Mechanical Engineering, State University of Campinas, Campinas, Brazil, 2010. 